\title{
Predictive Power Control of Wireless Sensor Networks for Closed Loop Control
}

\author{
Daniel E. Quevedo ${ }^{\dagger}$ and Anders Ahlén ${ }^{\ddagger}$ and Graham C. Goodwin ${ }^{\dagger}$ \\ $\dagger$ School of Electrical Engineering and Computer Science, The University of \\ Newcastle, NSW 2308, Australia dquevedo@ieee.org, \\ graham.goodwin@ newcastle.edu.au \\ $\ddagger$ Department of Engineering Sciences, Signals and Systems, Uppsala University, PO \\ Box 534 SE-751 21, Uppsala, Sweden; Anders. Ahlen@ signal.uu.se
}

Keywords : Wireless Sensor Networks, Predictive Control

We study a networked control architecture where wireless sensors are used to measure and transmit plant outputs to a remote controller. Packet loss probabilities depend upon the time-varying communication channel gains and the transmission powers of the sensors. Within this context, we develop a centralized stochastic nonlinear model predictive controller. It determines the sensor power levels by trading energy expenditure for expected plant state variance. To further preserve sensor energies, the power controller sends coarsely quantized power increment commands only when necessary. Simulations on measured channel data illustrate the performance achieved by the proposed controller.

\section{Introduction}

Wireless Sensor Networks (WSNs) are becoming an interesting alternative for closed loop control [1, 2]. WSNs can be placed where wires cannot go and where power sockets are not available. A drawback of using WSNs is that channel fading and interference may lead to packet errors and, thus, performance degradation. Whilst communication reliability and, thus, control accuracy, can certainly be improved by increasing transmission power levels, saving energy in WSNs is uppermost to avoid unnecessary maintenance, such as the replacement of batteries, see also [3, 4].

In the present work we examine a Networked Control System (NCS) architecture where sensor measurements are sent over wireless fading channels. In contrast to other approaches, see, e.g., [5], in the topology studied here, sensors do not communicate with each other. Instead, sensor measurements are sent to a single gateway for state estimation and subsequent plant input calculation. In addition, the gateway decides upon the power levels to be used by the sensors. Within this setting, we show how the sensor power levels can be designed via nonlinear predictive control. The proposed controller trades expected plant state variance for energy expenditure. The present work extends our recent conference contribution [6] (on state estimation) to NCS's.

\section{WSNs for Networked Control}

We consider an LTI $n$-dimensional plant with input $\{\mu(k)\}_{k \in \mathbb{N}_{0}}$ :

$$
x(k+1)=A x(k)+B \mu(k)+w(k), \quad k \in \mathbb{N}_{0} \triangleq\{0,1, \ldots\},
$$


where the initial state is Gaussian distributed with mean $x_{0}$ and covariance $P_{0} \in \mathbb{R}^{n \times n}$, i.e., $x(0) \in \mathcal{N}\left(x_{0}, P_{0}\right)$. Similarly, the driving noise process $w=\{w(k)\}_{k \in N_{0}}$ is i.i.d., where each $w(k) \in \mathcal{N}(0, Q)$.

A collection of $M$ sensors is used to measure and transmit plant output information via wireless links to a single gateway. Each sensor provides a noisy measurement signal, say $\left\{y_{m}(k)\right\}_{k \in N_{0}}$ :

$$
y_{m}(k)=C_{m} x(k)+v_{m}(k), \quad m \in\{1,2, \ldots, M\},
$$

and where $v=\left\{v_{m}(k)\right\}_{k \in N_{0}}$ is an i.i.d. process with $v_{m}(k) \in \mathcal{N}\left(0, R_{m}\right){ }^{1}$

The signals received at the gateway are then used to calculate the control input $\mu$. The aim is to steer the system state $x(0)$ to the origin. In the present work, we will assume that the associated control policy has already been designed and is given by linear state estimate feedback:

$$
\mu(k)=-L(k) \hat{x}(k), \quad k \in \mathbb{N}_{0} \triangleq\{0,1, \ldots\},
$$

where $\hat{x}(k)$ is an estimate of $x(k)$ and where $L(k)$ are given state feedback matrices of appropriate dimensions. Thus, the gateway needs to remotely estimate the state of the system (1). The situation is depicted in Fig. 1 for a networked control system (NCS) having $M=2$ wireless sensors.

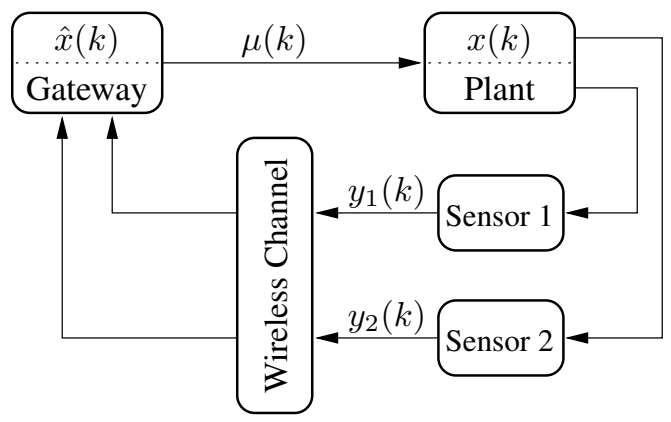

Figure 1: Networked Control with a WSN having $M=2$ sensors.

The distinguishing aspect of the situation at hand is that, since the $M$ links between sensors and gateway are wireless, transmission errors are likely to occur. This leads to loss of packets and control performance degradation. ${ }^{2}$ Packet loss probabilities depend upon the time-varying channel gains and upon the transmission power used by the sensors, higher power providing less transmission errors. However, in wireless sensor networks, it is of fundamental importance to save energy: Sensor nodes are expected to be operational for several years without maintenance. Thus, available energy resources have to be used with care. The main purpose of the present work lies in designing a centralized predictive power controller for the WSN used in the NCS of Fig. 1. Before presenting our proposal, we will first set the work in context by briefly elucidating the trade-off between power use and control accuracy.

\footnotetext{
${ }^{1}$ In addition to measurement noise, $v$ may also describe quantization effects.

${ }^{2}$ We will assume that sensor data is not affected by delays. Extensions to include time-delay issues, and also irregular sampling, does not present conceptual difficulties, provided sensor data are time-stamped.
} 


\section{Trading Energy Use for Control Performance}

To describe the interplay between energy consumption and transmission reliability, we quantify the energy used by each sensor $m$ at time $k \in \mathbb{N}_{0}$ via:

$$
g_{m}\left(u_{m}(k)\right) \triangleq \begin{cases}\frac{u_{m}(k) b_{m}}{r}+E_{\mathrm{P}} & \text { if } u_{m}(k)>0 \\ 0 & \text { if } u_{m}(k)=0 .\end{cases}
$$

Here, $u_{m}(k)$ is the transmission power used by the $m$-th sensor radio power amplifier, $b_{m}$ is the number of bits used per measurement value $y_{m}(k), r$ is bit-rate of the channels and $E_{\mathrm{P}}$ is the total energy needed (per measurement value) for power-up, sensing and circuitry. As we will see in Section 5, the choice of $b_{m}$ depends on the required accuracy and the energy available. A large value of $b_{m}$ will lead to an improved accuracy, but at the expense of a higher energy expenditure and an increased probability of packet error. In general, the number of bits per transmitted packet will be governed by the protocol used. In the present work, we focus on a simple scheme, where one measurement is transmitted at a time. ${ }^{3}$ The selected bit rate, $r$, will thus depend on the application as will the number of channels used.

Due to physical limitations of the radio power amplifiers, we assume that the power levels are constrained in magnitude according to:

$$
0 \leq u_{m}(k) \leq u_{m}^{\max }, \quad \forall k \in \mathbb{N}_{0}, \quad \forall m \in\{1,2, \ldots, M\}
$$

for given values $\left\{u_{m}^{\max }\right\}$. Thus, the maximum transmission energy per measurement value at each node is given by:

$$
\left(E_{\mathrm{TX}}^{\max }\right)_{m} \triangleq\left(b_{m} / r\right) u_{m}^{\max }, \quad m \in\{1,2, \ldots, M\} .
$$

We model transmission effects by introducing the $M$ binary stochastic arrival processes $\left\{\gamma_{m}(k)\right\}_{k \in N_{0}}, m \in\{1,2, \ldots, M\}$, where:

$$
\gamma_{m}(k)= \begin{cases}1 & \text { if } y_{m}(k) \text { arrives error-free at time } k \\ 0 & \text { if } y_{m}(k) \text { does not arrive error-free at time } k\end{cases}
$$

The associated success probabilities satisfy

$$
\mathcal{P}\left\{\gamma_{m}(k)=1\right\}=f_{m}\left(u_{m}(k) h_{m}(k)\right), \quad m \in\{1,2, \ldots, M\},
$$

where $f_{m}(\cdot):[0, \infty) \rightarrow[0,1]$ is a monotonically increasing function, which depends upon the communication scheme employed, and where $h_{m}(k)$ denotes the square of the magnitude of the channel gain.

To calculate the plant input $\mu(k)$ in (3), the gateway needs to obtain plant state estimates. Here, we will assume that the data transmitted incorporates error detection coding [7]. Hence, at any time $k$, past and present realizations of the transmission processes (7), say

$$
\gamma^{k} \triangleq \bigcup_{m \in\{1,2, \ldots, M\}}\left\{\gamma_{m}(0), \gamma_{m}(1), \ldots, \gamma_{m}(k)\right\}
$$

\footnotetext{
${ }^{3}$ Alternatively, one could also aggregate measurements.
} 
are available at the gateway. Faulty packets will be discarded when estimating the system state. This amounts to sampling (1)-(2) only at the successful transmission instants of each sensor link. Indeed, the conditional probability distribution of the system state at any time $k$, given $x_{0}, P_{0}, \gamma^{k}$ and correctly received sensor measurements up to time $k$, say $y^{k}$, is Gaussian. The conditional mean and covariance ${ }^{4}$ of the state, i.e.,

$$
\begin{aligned}
& \hat{x}(k) \triangleq \mathcal{E}_{w, v, x(0)}\left\{x(k) \mid y^{k}, \gamma^{k}\right\} \\
& \bar{P}(k) \triangleq \mathcal{E}_{w, v, x(0)}\left\{(\hat{x}(k)-x(k))(\hat{x}(k)-x(k))^{T} \mid y^{k}, \gamma^{k}\right\},
\end{aligned}
$$

satisfy the Kalman Filter recursions (see, e.g., [8]):

$$
\begin{aligned}
\hat{x}(k+1) & =A \hat{x}(k)+B \mu(k)+K(k+1)(y(k+1)-C(k+1)(A \hat{x}(k)+B \mu(k))) \\
P(k+1) & =A P(k) A^{T}+Q-A K(k) C(k) P(k) A^{T} \\
\bar{P}(k) & =P(k)-K(k) C(k) P(k),
\end{aligned}
$$

with initial values $P(0)=P_{0}$ and $\hat{x}(0)=x_{0}$ and where: ${ }^{5}$

$$
\begin{aligned}
& C(k) \triangleq\left[\begin{array}{llll}
\gamma_{1}(k)\left(C_{1}\right)^{T} & \gamma_{2}(k)\left(C_{2}\right)^{T} \quad \ldots \quad \gamma_{M}(k)\left(C_{M}\right)^{T}
\end{array}\right]^{T} \\
& K(k) \triangleq P(k) C(k)^{T}\left(C(k) P(k) C(k)^{T}+R\right)^{-1} \\
& R \triangleq \operatorname{diag}\left(R_{1}, R_{2}, \ldots, R_{M}\right) \text {. }
\end{aligned}
$$

The state estimate in (10) is used to calculate the plant input, see (3). The controlled plant (1)-(3) is, thus, described via:

$$
x(k+1)=\bar{A}(k) x(k)+e(k)+w(k),
$$

where $\bar{A}(k) \triangleq A-B L(k)$, whereas

$$
e(k) \triangleq B L(k)(x(k)-\hat{x}(k))
$$

denotes the effect of the state estimation error on the successor plant state.

We note that $e(k)$ in (12) depends upon the transmission processes $\gamma_{m}(k)$ through the matrices $C(k)$ in the state estimate $\hat{x}(k)$, see (10) and (11). As seen in (8), transmission reliability can be improved by using larger power levels $u_{m}(k)$, however, this occurs at the expense of more energy consumption, see (4). This trade-off between energy consumption at the sensors and resulting control accuracy forms the background to the power control scheme proposed in the following section.

\section{Predictive Power Control}

In the NCS architecture under study, the gateway not only calculates the plant inputs, but also determines the power levels to be used by the sensors. For that purpose, the gateway is equipped with a model predictive controller which trades energy consumption for control quality over a future prediction horizon. Power control signals are sent over wireless links to the sensors. ${ }^{6}$

\footnotetext{
${ }^{4}$ Here, $\mathcal{E}_{w, v, x(0)}$ denotes expectation taken w.r.t. the noise sequences $w$ and $v$ and the initial state $x(0)$.

${ }^{5}$ Properties of this (and related) estimators have been studied, e.g., in [9, 10, 11].

${ }^{6}$ At the gateway saving energy is of less importance than at the sensors. We, thus, assume that communication from the gateway to the sensors is error-free.
} 
Power Control Signal Coding To keep processing and associated power consumption at the sensors to a minimum, in our approach the power control signals have short word-lengths. Here, we will use coding ideas frequently used in power control architectures for cellular networks, see, e.g., [12] (and compare also to our work on NCS's in [13]) and send coarsely quantized power increments, say $\Delta u_{m}(k)$, rather than actual power values, $u_{m}(k)$, to each sensor $m \in\{1,2, \ldots, M\}$. We thus have:

$$
\Delta u_{m}(k) \in \mathbb{U}_{m}, \quad \forall k \in \mathbb{N}_{0}, \quad \forall m \in\{1,2, \ldots, M\},
$$

where $\left\{\mathbb{U}_{m}\right\}$ are given finite sets, each having a small number of elements.

Upon reception of $\Delta u_{m}(k)$, each sensor $m$ reconstructs the power level to be used by its radio power amplifier by simply setting

$$
u_{m}(k)=u_{m}(k-1)+\Delta u_{m}(k) .
$$

For further reference, we define the signal

$$
\Delta u(k) \triangleq\left[\begin{array}{lll}
\Delta u_{1}(k) & \ldots & \Delta u_{M}(k)
\end{array}\right]^{T}, \quad k \in \mathbb{N}_{0}
$$

and note that the quantization constraint (13) imposes:

$$
\Delta u(k) \in \mathbb{U} \triangleq \mathbb{U}_{1} \times \mathbb{U}_{2} \times \cdots \times \mathbb{U}_{M}, \quad \forall k \in \mathbb{N}_{0} .
$$

Predictive Power Controller At every time instant $k \in \mathbb{N}_{0}$, the predictive power controller first calculates $\bar{P}(k)$, which results from iterating (10) for the (known) past arrival process realizations $\gamma^{k}$, see (9). It also obtains channel gain predictions over a finite horizon of fixed length $N$, namely:

$$
\left\{\hat{h}_{m}(k+1 \mid k), \hat{h}_{m}(k+2 \mid k), \ldots, \hat{h}_{m}(k+N \mid k)\right\}, \quad \forall m \in\{1,2, \ldots, M\},
$$

which can be estimated by using previous channel estimates, see, e.g., [14, 15]. Given this information, the controller minimizes the finite-set constrained cost ${ }^{7}$

$$
J(\Delta U) \triangleq \sum_{\ell=k+1}^{k+N}\left\{\mathcal{E}_{\Gamma(k)}\left\{\operatorname{trace}\left(\Sigma^{\prime}(\ell)\right)\right\}+\rho \sum_{m=1}^{M} g_{m}\left(u_{m}^{\prime}(\ell)\right)\right\},
$$

where $g_{m}\left(u_{m}^{\prime}(\ell)\right)$ is as in (4) and where

$$
\begin{aligned}
\Sigma^{\prime}(\ell) & \triangleq \mathcal{E}_{w, v, x(0)}\left\{e^{\prime}(\ell) e^{\prime}(\ell)^{T} \mid y^{k}, \gamma^{k}\right\}=B L(\ell) \bar{P}^{\prime}(\ell) L(\ell)^{T} B^{T}, \\
\Delta U & \triangleq\left\{\Delta u^{\prime}(k+1), \Delta u^{\prime}(k+2), \ldots, \Delta u^{\prime}(k+N)\right\},
\end{aligned}
$$

see (10) and (12). The scalar $\rho \geq 0$ is a design parameter which allows one to trade control accuracy for energy consumption. The expectation operator $\mathcal{E}_{\Gamma(k)}$ is taken with respect to the distribution of future transmission outcomes in ${ }^{8}$

$$
\Gamma(k) \triangleq\left[\begin{array}{llll}
\gamma(k+1) & \gamma(k+2) & \ldots & \gamma(k+N)
\end{array}\right]^{T} .
$$

This distribution depends upon the power level, see (8). The decision variables, i.e., the tentative future power value increments, are collected in $\Delta U$, see (15) and (17). These determine the tentative future power levels $u_{m}^{\prime}(\ell)$ in (16) via

$$
\begin{aligned}
& u_{m}^{\prime}(\ell)=u_{m}^{\prime}(\ell-1)+\Delta u_{m}^{\prime}(\ell), \quad \ell \in\{k+1, \ldots, k+N\}, m \in\{1,2, \ldots, M\}, \\
& { }^{7} \text { Primed variables refer to tentative values of the corresponding physical variables. } \\
& { }^{8} \text { Compare to the scenario based approaches taken in [16]. }
\end{aligned}
$$

\footnotetext{
${ }^{7}$ Primed variables refer to tentative values of the corresponding physical variables.
} 
starting from the current values, $u_{m}^{\prime}(k)=u_{m}(k), \forall m \in\{1,2, \ldots, M\}$.

Minimization of (16) subject to the constraints:

$$
\begin{gathered}
\Delta U \in \mathbb{U}^{N} \triangleq \mathbb{U} \times \mathbb{U} \times \cdots \times \mathbb{U} \\
0 \leq u_{m}^{\prime}(\ell) \leq u_{m}^{\max }, \quad \forall \ell \in\{k+1, \ldots, k+N\}, \forall m \in\{1,2, \ldots, M\}
\end{gathered}
$$

gives the sequence of control increments:

$$
\Delta U^{\mathrm{opt}} \triangleq \arg \min J(\Delta U)
$$

Following the moving horizon principle, only the $M$ power updates in 9

$$
\Delta u(k+1)^{\mathrm{opt}} \triangleq\left[\begin{array}{llll}
I_{M} & 0_{M} & \ldots & 0_{M}
\end{array}\right] \Delta U^{\mathrm{opt}}
$$

are sent to the corresponding sensors. At the next time step, namely $k+1$, the optimization procedure is repeated, giving rise to power level increments $\Delta u(k+2)^{\text {opt }}$. This procedure is repeated ad infinitum.

We emphasize that, despite (18) being a stochastic nonlinear programme, $\Delta U^{\text {opt }}$ can be found via simple exhaustive search over the $2^{M N}$ possible transmission scenarios $\Gamma(k)$ and, in a worst complexity case, $|\mathbb{U}|^{N}$ values of $\Delta U$.

The proposed controller jointly decides upon the power levels of all $M$ sensors to achieve the best trade-off between energy use and control accuracy.

\section{Simulation Study}

To illustrate basic features of the model predictive power controller presented in the previous section, we consider a NCS as in Fig. 1 with $M=2$ channels and use measured channel data. Measurements were acquired in the $2.4 \mathrm{GHz}$ ISM band in an office area at the Signals and Systems group at Uppsala University.

We assume that Binary Phase Shift Keying is employed and use the transmission error model [7]:

$$
f_{m}\left(u_{m}(k) h_{m}(k)\right)= \begin{cases}\left(1-\tilde{Q}\left(\sqrt{2 u_{m}(k) h_{m}(k) /\left(r k_{B} T\right)}\right)\right)^{b_{m}} & \text { if } u_{m}(k)>0 \\ 0 & \text { if } u_{m}(k)=0\end{cases}
$$

where $\tilde{Q}(z) \triangleq 1 / \sqrt{2 \pi} \int_{z}^{\infty} \exp \left(-\nu^{2} / 2\right) d \nu$ is the Q-function, $k_{B}$ is the Boltzmann constant and $T=300[\tilde{K}]$ is the temperature. The selected bit-rate of the channels is $r=40[\mathrm{kbits} / \mathrm{s}]$, and the number of bits per measurement value are $b_{1}=b_{2}=$ 8 [bits].

The (unstable) plant is characterized via the model of Section 2 with:

$A=\left[\begin{array}{ll}2 & 1 \\ 1 & 1\end{array}\right], B=\left[\begin{array}{l}1 \\ 1\end{array}\right], x_{0}=\left[\begin{array}{l}0 \\ 0\end{array}\right], P_{0}=\frac{1}{3} I_{2}, Q=\frac{1}{2} I_{2},\left[\begin{array}{l}C_{1} \\ C_{2}\end{array}\right]=I_{2}, R_{1}=R_{2}=\frac{1}{100}$.

The plant input in (3) is provided by the LQG policy: $\mu(k)=-\left[\begin{array}{ll}1.452 & 0.898\end{array}\right] \hat{x}(k)$.

The constraints on the power values, see (5), are $u_{1}^{\max }=u_{2}^{\max }=0.5[\mathrm{~mW}]$. The power controller parameters are chosen as $N=1, \rho=10[1 / \mu J]$. Power increments are restricted to belong to the finite sets $\mathbb{U}_{1}=\mathbb{U}_{2}=\{0, \pm 50\}[\mu W]$. We assume that the gateway has perfect one-step-ahead channel predictions.

${ }^{9} I_{M}$ denotes the $M \times M$ identity matrix and $0_{M}$ the all zeros $M \times M$ matrix. 
To investigate the impact of different kinds of sensor nodes, we introduce the energy ratio $\eta \triangleq E_{\mathrm{P}} / E_{\mathrm{TX}}^{\max }$, see (4) and (6).

Fig. 2 illustrates that the proposed predictive controller tries to find the best compromise between the two sensor links. In particular, the controller at times approximately inverts the channel gains. At other times, it decides to send one of the sensors to sleep, i.e., to set $u_{1}(k)=0$ or $u_{2}(k)=0$. This scheduling aspect is even more apparent in Fig. 3, as a consequence of a higher value of $\eta$. It is important to notice that, in the present case, the first component of $x(k)$ is more important than the second component. Consequently, the predictive controller favors Sensor 1 over Sensor 2.

If power levels would be kept constant, such that the same total amount of energy is used, then, for $\eta=0.5$, the trace of the empirical covariance of the state would be $25 \%$ larger. For $\eta=1.7$, no such constant power levels exist, since $E_{\mathrm{P}}$ is too large.
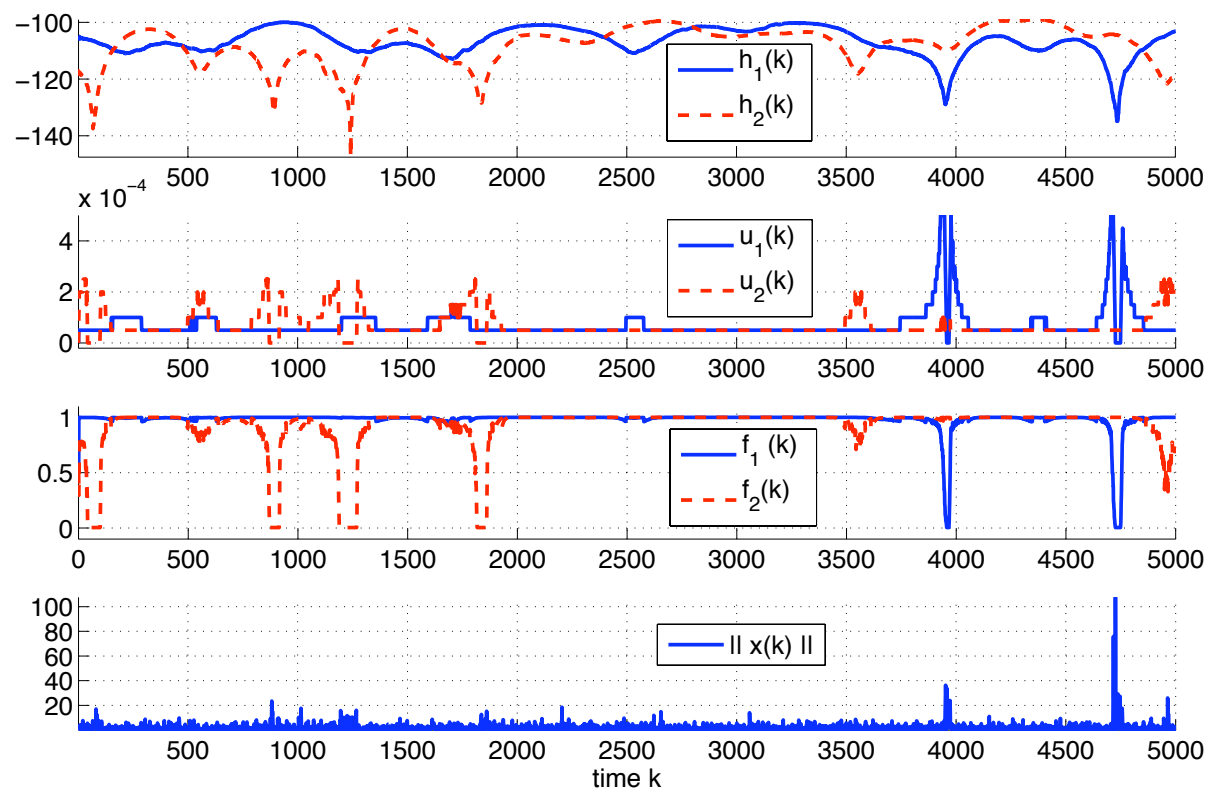

Figure 2: System performance for sensor nodes with energy ratio $\eta=0.5$.

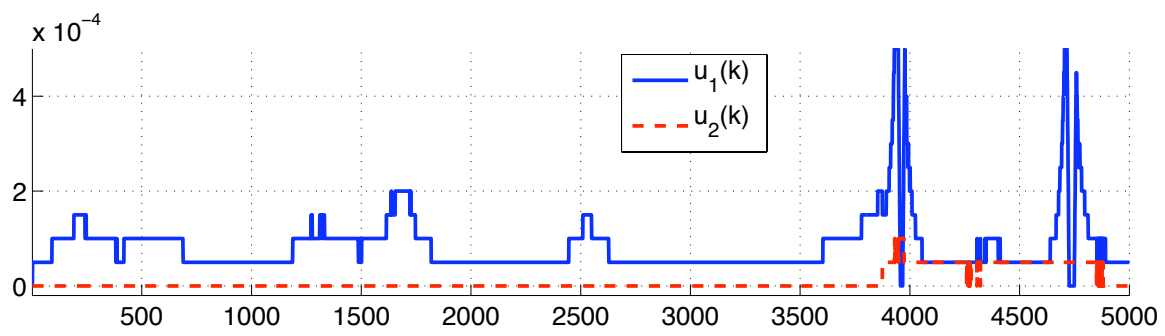

Figure 3: Power levels for sensor nodes with energy ratio $\eta=1.7$. 


\section{Conclusions}

We have developed a stochastic nonlinear model predictive power controller for wireless sensor networks used within a networked control system. Due to (time-varying) fading on the wireless channels, transmission errors of sensor measurements are likely to occur. The proposed controller trades energy expenditure at the sensors for expected plant state variance to compensate for channel fading in an optimal manner, as illustrated on measured channel data. Key to keeping the computational burden limited is the fact that the occurrence of transmission errors constitutes a binary random variable. Thus, expectations can be exactly evaluated via finite sums, i.e., no integrals need to be evaluated or approximated.

\section{References}

[1] P. Antsaklis and J. Baillieul, "Special issue on technology of networked control systems," Proc. IEEE, vol. 95, pp. 5-8, Jan. 2007.

[2] X. Shen, Q. Zhang, and R. Caiming Qiu, "Wireless sensor networking [guest ed.]," IEEE Wireless Commun., vol. 14, pp. 4-5, Dec. 2007.

[3] J.-J. Xiao, A. Ribeiro, Z.-Q. Luo, and G. B. Giannakis, "Distributed compressionestimation using wireless sensor networks," IEEE Trans. Signal Processing, vol. 23, pp. 27-41, July 2006.

[4] X. Luo and G. B. Giannakis, "Energy-constrained optimal quantization for wireless sensor networks," EURASIP J. Adv. Signal Processing, 2008.

[5] A. Speranzon, C. Fischione, K. H. Johansson, and A. Sangiovanni-Vincentelli, "A distributed minimum variance estimator for sensor networks," IEEE J. Select. Areas Commun., vol. 26, pp. 609-621, May 2008.

[6] D. E. Quevedo and A. Ahlén, "A predictive power control scheme for energy efficient state estimation via wireless sensor networks," in Proc. IEEE Conf. Decis. Contr., 2008.

[7] J. G. Proakis, Digital Communications. New York, N.Y.: McGraw-Hill, 3rd ed., 1995.

[8] B. D. O. Anderson and J. Moore, Optimal Filtering. Prentice Hall, 1979.

[9] B. Sinopoli, L. Schenato, M. Franceschetti, K. Poolla, M. I. Jordan, and S. S. Sastry, "Kalman filtering with intermittent observations," IEEE Trans. Automat. Contr., vol. 49, pp. 1453-1464, Sept. 2004.

[10] M. Huang and S. Dey, "Stability of Kalman filtering with Markovian packet losses," Automatica, vol. 43, pp. 598-607, 2007.

[11] X. Liu and A. Goldsmith, "Kalman filtering with partial observation losses," in Proc. IEEE Conf. Decis. Contr., (Paradise Island, Bahamas), pp. 4180-4186, 2004.

[12] H. Holma and A. Toskala, eds., WCDMA for UMTS. West Sussex, England: John Wiley \& Sons, third ed., 2004.

[13] G. C. Goodwin, H. Haimovich, D. E. Quevedo, and J. S. Welsh, "A moving horizon approach to networked control system design," IEEE Trans. Automat. Contr., vol. 49, pp. 1427-1445, Sept. 2004.

[14] T. Ekman, M. Sternad, and A. Ahlén, "Unbiased power prediction of Rayleigh fading channels," in Proc. Vehicular Technology Conf., vol. 1, pp. 280-284, 2002.

[15] T. Ekman, Prediction of Mobile Radio Channels: Modeling and Design. PhD thesis, Uppsala University, Oct. 2002.

[16] D. E. Quevedo, E. I. Silva, and G. C. Goodwin, "Control over unreliable networks affected by packet erasures and variable transmission delays," IEEE J. Select. Areas Commun., vol. 26, pp. 672-685, May 2008. 